
internationales

vol. 20 - n² 2004

L'asile politique en Europe depuis l'entre-deux-guerres

\title{
Tirer l'échelle ? Tensions et rejets de nouveaux arrivants au sein de l'émigration antifasciste italienne
}

Éric Vial

\section{(2) OpenEdition}

Journals

Édition électronique

URL : https://journals.openedition.org/remi/965

DOI : $10.4000 /$ remi.965

ISSN : $1777-5418$

Éditeur

Université de Poitiers

Édition imprimée

Date de publication : 1 septembre 2004

Pagination : 39-56

ISBN : 2-911627-37-7

ISSN : 0765-0752

Référence électronique

Éric Vial, «Tirer l'échelle ? Tensions et rejets de nouveaux arrivants au sein de l'émigration antifasciste italienne », Revue européenne des migrations internationales [En ligne], vol. $20-n^{\circ} 2$ | 2004, mis en ligne le 25 septembre 2008, consulté le 16 avril 2022. URL : http://journals.openedition.org/remi/965 ; DOI : https://doi.org/10.4000/remi.965

Ce document a été généré automatiquement le 16 avril 2022.

(c) Université de Poitiers 


\title{
Tirer l'échelle? Tensions et rejets de nouveaux arrivants au sein de l'émigration antifasciste italienne
}

\author{
Éric Vial
}

1 Du point de vue du pays d'accueil, tout groupe immigré tend à être présenté comme compact, ne serait-ce qu'en fonction des mêmes facilités langagières qui font parler de «communautés ». En même temps, des clivages géographiques sont manifestes, à commencer, dans le cas italien, par celui traditionnel entre Nord et Sud. Les clivages idéologiques ne le sont pas moins, surtout quand se juxtaposent migrants économiques et politiques, opposants exilés et représentants du pays d'origine venus encadrer leurs compatriotes au nom d'une Église ou directement de l'État. S'y ajoutent des tensions lorsque de nouveaux venus sont rejetés par les éléments plus anciennement installés, comme l'a observé Pierre Milza pour les Italiens arrivés à Chambéry après 1945 (Milza, $\left.1988: 2^{\circ} 108\right)$. Au sein d'une émigration politique, ces tensions ne sont guère prises en compte, éclipsées par les débats idéologiques ou les concurrences entre organisations. Or elles pèsent là aussi.

2 C'est le cas pour l'émigration antifasciste italienne dans la France de l'entre-deuxguerres. (Garosci, 1953 ; Droz, 1958 ; Milza, 1985 ; Tombaccini, 1988). Cette émigration est très composite, de quelques libéraux et catholiques à des anarchistes, en passant par quelques anarcho-syndicalistes, des républicains mazziniens, les «libérauxsocialistes » de Giustizia e Libertà, des socialistes réformistes ou maximalistes. Elle bruit de débats et de polémiques, encore que les divisions apparentes soient atténuées par des sociabilités interpersonnelles et des tentatives unitaires, avec des organisations transversales comme la LIDU, Ligue italienne des Droits de l'Homme (Vial, 1998), ou, de 1937 à la guerre, l'Union Populaire italienne, satellite du PCI, Parti Communiste Italien (Rapone, 1986; Vial, 1999), mais aussi avec des regroupements, la Concentration antifasciste rassemblant les principales organisations non communistes de 1927 à 1934, des esquisses de front populaire ensuite. Elle est aussi caractérisée par des phénomènes de politisation et de dépolitisation qui, conjugués à l'importance de l'émigration 
transalpine en France, près d'un million de personnes en 1930, impliquent une forte interpénétration entre émigration politique et économique (Milza, 1988; Témime, 1991 ; Vial, 1988). D'où l'importance des contacts avec les émigrés déjà installés, mais aussi de l'accueil des nouveaux venus. Or celui-ci pose problème, y compris lorsqu'il s'agit de gens qui ont fui le régime fasciste, et on constate des grincements, en particulier autour de trois moments-clé, 1930-1931, 1937-1938 et, en remontant dans le temps, 1926-1927.

\section{0-1931 : le rejet d'une émigration économique}

On rencontre le prototype de la réaction de rejet de nouveaux arrivants en 1930. Si la France d'alors n'a pas tout à fait conscience d'être touchée par la crise mondiale, l'Italie la subit de façon directe; le 13 août, après trois ans de fermeture des frontières, Mussolini les rouvre. Là où a commencé une agitation sociale et potentiellement politique, les préfets doivent accorder des passeports et ne plus guère se soucier de l'émigration clandestine, ce qui fait penser aux autorités française que celle-ci est organisée plus même que tolérée (Sori, 1979: 436-437)1. Très vite, le journal des maximalistes affirme que les douaniers italiens indiquent les passages aux partants dépourvus de papiers en règle ${ }^{2}$. Mais dans un premier temps, côté français, on craint surtout l'introduction d'espions dans les zones d'édification de fortifications ${ }^{3}$. Un autre aspect inquiète : devant les sénateurs du royaume, Mussolini a présenté l'ouverture des frontières comme "une mesure morale destinée à démontrer aux mécontents, à travers l'expérience directe, qu'en ce moment il n'existe pas de pays facile dans aucune partie du monde ». Ils doivent comprendre la leçon, revenir à la mère-patrie (Sori, 1979 : 436). De notre côté des Alpes, il se dit qu'il s'agit de ternir l'image de la France en exploitant le mécontentement des clandestins soumis à un refoulement. Président de la LIDU, organisation unitaire de l'émigration non communiste vouée à l'assistance aux émigrés et ayant plus ou moins l'oreille des autorités françaises Luigi Campolonghi partage ce point de vue, développé dans différents journaux d'organisations en exil. Début 1931, il lance une campagne pour souligner que le fascisme est seul responsable de la situation pour avoir interdit les départs quand la France demandait de la main d'œuvre et les autoriser quand toutes les places sont prises ${ }^{4}$.

4 La Ligue cherche à canaliser l'afflux. Elle multiplie les avertissements, répète qu'il ne faut pas venir à Paris, à moins d'avoir déjà un contrat de travail, discours tenu aussi à titre privé à Carlo Rosselli, leader de Giustizia e Libertà, par le secrétaire de la LIDU, Alceste De Ambris : se diriger vers Paris revient à se condamner à mourir de faim ${ }^{5}$. Sans doute par impuissance, les menaces se mêlent aux avertissements. Ceux qui viendront ne pourront être aidés, on les considèrera "non comme des réfugiés dans le besoin, mais comme des ouvriers qui changent volontairement de résidence». Il en va de même à Grenoble, Lyon ou Marseille, destinations traditionnelles; en revanche, des départements sont conseillés, il y reste des emplois à pourvoir: Ardennes, Gironde, Loire, Meurthe-et-Moselle, Vienne, Var, Moselle, Gard, Maine-et-Loire, Haut-Rhin, Belfort, Constantine ${ }^{6}$. Mais avec le temps cette liste se rétrécit, et on conseille de ne pas tenter de s'installer en France. La Ligue ne pourra faciliter la régularisation des clandestins si leur spécialité professionnelle n'est pas demandée là où ils arrivent, l'émigration sans contrat est fustigée comme incompatible avec le réalisme mais aussi avec la solidarité de classe, car elle gonfle les rangs des chômeurs, armée de réserve du 
capital. À l'automne 1931, quand la France ne peut plus se prendre pour une oasis protégée, un tel avertissement se trouve dans toute la presse de l'émigration, y compris celle des maximalistes ou des anarchistes?

5 Une très réelle hostilité se manifeste envers les nouveaux venus. Elle s'appuie sur la méfiance, avant l'ouverture de la frontière, envers les rares qui arrivaient avec un passeport en règle. On n'imaginait guère que certains passeraient à l'antifascisme, qui sympathisant du PCI, qui proche de la LIDU, qui volontaire en Espagne ${ }^{8}$. Encore la Libertà, l'hebdomadaire de la Concentration antifasciste avait noté qu'en Lorraine au moins, «à peine ont-ils respiré l'air libre [ils] se dépêchent de jeter aux orties les cartes et les insignes qui leur avaient été imposées "`. Mais le plus souvent, leur passeport les désignait comme des éléments sûrs pour le régime, étrangers au mouvement ouvrier, peu portés aux revendications, prêts à accepter des salaires insuffisants ou de mauvaises conditions de travail. D'où déjà des protestations contre "l'introduction de travailleurs inconscients, encadrés par des fascistes, sur le marché de l'emploi, dans certains centres comme Le Havre et Rouen $»^{10}$. Aussi, la vague de nouveaux venus ne peut être bien accueillie. Même les clandestins sont suspects, malgré une sollicitude a priori : ils peuvent être dépourvus de passeport pour donner le change, et à la LIDU, la section haut-savoyarde de Saint-Jeoire, par exemple, dit vouloir démasquer les fascistes cachés parmi eux. Cependant, en septembre 1930 encore, De Ambris proteste contre des refoulements et demande malgré des méfiances que les clandestins soient considérés comme des réfugiés politiques ${ }^{11}$.

6 En pratique, ces nouveaux venus sont des concurrents très tôt dénigrés. Selon les maximalistes, ils sont sans qualification, peu travailleurs, susceptibles de compromettre la bonne réputation de la main d'œuvre italienne ${ }^{12}$. Selon De Ambris ils font le jeu de Mussolini en quittant l'Italie au lieu d'y attendre « l'heure de la justice et de la révolution, que leur départ risque de retarder $»^{13}$. Selon un ancien député socialiste, Francesco Ciccotti, le fascisme a conseillé aux migrants de se faire passer pour des réfugiés et de s'adresser à la LIDU, et il annonce qu'à Nice seuls seront aidés ceux qui sont recommandés par des personnes de confiance, les autres pouvant s'adresser aux consulats ${ }^{14}$. Derrière tout cela, il y a le mépris de qui a fui l'Italie dès les premières années du régime pour ceux qui en ont subi plusieurs années le joug. Dès octobre 1930, revenu de ses générosités, De Ambris a écrit au responsable de la section grenobloise que ces gens ne sont réfugiés que "jusqu'à un certain point ». Ils n'auraient senti "l'intolérable honte du fascisme sinon lorsqu'ils ont eu l'estomac vide (...) du point de vue humain, ils sont dignes de pitié, mais du point de vue politique ils sont assez peu intéressants, sauf de rares exceptions ». Fin 1931, il leur reproche de ne pas s'être révoltés en Italie. Peut-être exorcise-t-il son échec à agir depuis la France, en fantasmant sur ce qu'il aurait pu faire à leur place... Son ami Giovanni Favaletto, responsable de la section de Nancy, parle avec mépris de "gens qui sont restés jusqu'à hier en Italie, avec la carte du syndicat fasciste dans leur poche $»^{15}$. Cette hostilité n'est pas l'apanage de la LIDU. Pour le PCI, l'ouverture de la frontière a pour but d'inonder la France d'espions ou d'offrir à des fascistes des voyages vers les lieux où il reste du travail ${ }^{16}$.

7 Reste que des voix s'élèvent contre une attitude incompatible avec les traditions et les idéaux de la LIDU. Certains responsables locaux, par exemple à Modane, restent partisans de l'aide à tous les nouveaux venus ${ }^{17}$. Le refus de distinguer entre ces derniers et les réfugiés anciens, ainsi qu'entre militants politiques et émigrés économiques, s'appuie sur le vieux leader réformiste Filippo Turati qui parle de migrants 
«économico-politiques » que le fascisme ne menace pas de prison mais empêche de travailler. Victimes du régime, ce sont bien des réfugiés politiques ${ }^{18}$. Ce discours est approuvé, mais passe peu dans la pratique, sauf chez ceux qui sont déjà convaincus. Ceux-ci se regroupent en particulier autour de l'Esilio, journal réunissant des antifascistes mal à leur aise dans leurs organisations. Plus sensibles aux situations individuelles qu'à la politique générale, ils refusent les arguments fondés sur le chômage car "mieux valent la faim et la liberté que la faim seulement", demandent à la LIDU de ne pas épouser les préoccupations égoïstes des Français, veulent que les nouveaux venus soient tenus non pour des fascistes mais pour des jeunes à faire adhérer ou de vieux militants qui rejoignent enfin leurs camarades. Ils craignent une transformation de l'antifascisme en «caste close». Ils trouvent un écho dans les sections de la LIDU, comme celle de Grenoble hostile à tout « refus de solidarité » car ne pas s'ouvrir aux nouveaux venus revient à s'interdire « de jouer quelque rôle que ce soit $»^{19}$.

Cette attitude est minoritaire, d'autant qu'avec la crise, la LIDU a de plus en plus de difficultés dans sa mission d'assistance, malgré le rêve jamais tout à fait abandonné de transformer les sections en consulats parallèles à ceux du régime ${ }^{20}$. Au congrès de 1931, l'idée d'aide matérielle même est remise en cause par De Ambris, et réservée aux cas extrêmes. Lors de celui de 1932, face à la multiplication des demandes d'intervention auprès des pouvoirs publics et à l'augmentation du pourcentage d'échecs, il s'en prend à ceux qui «ne sont absolument pas des réfugiés politiques mais seulement des émigrés pourvus d'un passeport en règle, expatriés pour des raisons purement économiques ", veut "économiser le peu de possibilités qui nous restent » et les réserver aux adhérents, encore que cela implique de préférer de nouveaux arrivants susceptibles de rejoindre l'organisation à ceux qui, installés, n'ont pas jugé bon de le faire ${ }^{21}$. En fait, il représente un courant soucieux de ne pas réduire l'organisation à l'assistance, d'en faire le porte-voix politique de ceux qui, comme lui, ne se reconnaissent pas dans les partis reconstitués. À l'inverse, Campolonghi, lié à la direction réformiste, se satisfait d'une fonction d'aide facilitant le recrutement, donne priorité aux militants mais sans exclusive, définit le réfugié comme celui "qui a dû abandonner l'Italie pour échapper à des condamnations politiques, à des persécutions certaines, ou pour obéir à un bannissement qui lui a été signifié par les fascistes » mais aussi celui «qui bien qu'étant parti d'Italie sans être personnellement menacé, a manifesté, une fois à l'étranger, son opposition ouverte au régime, ce qui, parce que cela constitue dans l'état présent de la législation fasciste un délit, l'exposerait à son retour en Italie à des condamnations ou à des représailles $»^{22}$.

Hésitations et contradictions donnent l'impression de tempêtes dans un verre d'eau, mais renvoient à une crise profonde, entre fatigue de l'exil face à un fascisme dont on se lasse d'attendre la chute, et difficultés économiques avec leur cortège de réactions xénophobes dans le pays dit d'accueil. L'émigration politique italienne non communiste voit obérer son élan et ses capacités de recrutement. Le rejet de nouveaux arrivants n'en constitue pas le point de départ, mais la conséquence de difficultés

et d'un essoufflement, il les accélère cependant. C'est bien un clivage entre "générations » séparées par un laps de temps fort bref, puisque le premier afflux a cessé en 1927, séparées surtout par des expériences différentes - sans doute aussi par le fait que les nouveaux venus sont des anonymes, sans légitimité politique. 


\section{7-1938: de la main tendue à l'accueil des victimes des lois raciales}

10 Il ne semble pas que des leçons soient tirées de cette expérience. Trop d'autres difficultés se sont accumulées, faisant écran. Le rejet des nouveaux venus apparaît comme un élément mineur. La coupure même entre émigrés politiques et économiques est absente tant des débats du moment que des souvenirs des témoins. Ce sont d'autres mécanismes qui au milieu de la décennie amènent le PCI à tenter d'adopter et de faire adopter une attitude inverse, d'abord de manière générale, ensuite face à aux arrivées liées aux lois antisémites mussoliniennes de 1938. Cela relève de l'adaptation de la tactique thorézienne de la main tendue, dans un souci d'unité nationale face au péril constitué par l'Allemagne nazie. Ceci quitte à accepter le fascisme ou au moins à tenter d'agir à l'intérieur des syndicats italiens officiels et à faire appel aux « frères en chemise noire $»^{23}$. En France, les grèves de juin 1936 ont amené à la CGT des inscrits jusque-là réputés fascistes ${ }^{24}$. Mais la guerre d'Espagne réveille l'antifascisme, et les autres organisations en exil, non communistes, ou des militants de base, voient d'un mauvais œil ce qui semble une compromission. Reste que ce discours, quelque peu édulcoré, sert de support à une ouverture aux nouveaux venus ou aux Italiens de passage, saisonniers, touristes. Arriver tout droit d'Italie ne devrait plus être dirimant.

11 Ainsi, en mars 1937 est créée l'UPI, Union Populaire Italienne, organisation de masse du PCI destinée à réunir le plus grand nombre possible d'émigrés, antifascistes mais surtout apolitiques et appartenant aux "masses influencées par le fascisme ", au nom de "l'union du peuple italien » autour du triptyque du front populaire français, pain, paix et liberté. Dès le congrès constitutif, il est demandé aux inscrits de chercher cette union "en approchant et en parlant avec tous les éléments qui vont et viennent de l'Italie "25. Il s'agit entre autres de répondre aux critiques du Komintern, qui exige une action vers le pays d'origine (Urban 1986: 142) ${ }^{26}$, en s'intéressant aux Italiens de passage, saisonniers ou touristes, et c'est une des fonctions du quotidien de l'UPI, la Voce degli italiani, lancé en juin $1937^{27}$. On insiste sur l'Exposition universelle de Paris; Lourdes jouerait le même rôle, mais l'UPI, parisianocentriste et peu implantée dans le Sud-ouest, a aussi quelque mal à concilier la main tendue avec la tradition anticléricale italienne. Toujours est-il qu'à côté d'une émigration officielle, limitée mais non tarie, le tourisme couvre des expatriations. Les adhérents à l'UPI sont priés de faire bon accueil aux nouveaux venus. La direction dénonce l'hostilité à leur égard. La Voce parle de «nouvelles catégories de réfugiés politiques", affirme que dans chaque train arrivant d'Italie à Paris, de 80 à 90 passagers ne repartent pas, souvent pour ne pas être envoyées en Espagne combattre pour Franco. Certes, on retrouve les préventions de 1930, et l'UPI explique que mieux vaudrait organiser l'agitation en Italie plutôt que de déserter, mais elle juge aussi nécessaire d'aider tout compatriote dont l'attitude est contraire aux intérêts du fascisme ${ }^{28}$. Elle recommande d'approcher des Italiens venant en France et favorables au régime, de leur montrer ses tares, de « leur parler fraternellement sans offenser en rien leurs sentiments », de leur faire lire le quotidien, et cite un visiteur qui, après avoir accepté de le faire, aurait donné cinq cent francs ${ }^{29}-$ entre exemple édifiant pour convaincre le lecteur, et souci de faire passer pour un don individuel une partie des subventions soviétiques, vertueusement niées. Le journal donne par ailleurs en exemple des Italiens de Guéret, dans la Creuse, qui, fraîchement arrivés de la péninsule, ont déjà pu 
constater la différence entre CGT et syndicats fascistes, ou tel autre, arrivé carte du fascio en poche et qui se rend compte qu'il a été trompée ${ }^{30}$.

12 L'effort vers ces nouveaux venus est un thème récurrent du journal, avec tel jeune présent en France depuis trois mois, tel vieux Sicilien installé depuis le même laps de temps à Molières, dans le Gard, ou tels émigrés récents, sans autre précision, à Argenteuil $^{31}$. Mais l'insistance même peut mettre la puce à l'oreille. D'autant que l'on n'est pas dans une période de grand afflux, ces nouveaux venus ne sont pas un objectif essentiel et l'UPI vise plutôt la conquête d'éléments installés en France mais fascistes ou réputés tels. On pourrait ajouter les éléments jusque là apolitiques. Une vieille émigration méridionale, souvent dépourvue de traditions d'engagement, fait aussi partie des objectifs, sans grand résultat, peut-être du fait d'une longue incurie des militants de tous partis ${ }^{32}$. Les nouveaux venus sont noyés dans ces préoccupations, mais il en est question assez régulièrement. On peut imaginer une perpétuation de la tendance des émigrés à tirer l'échelle derrière eux, à souhaiter avoir plus à voir avec les autochtones qu'avec les derniers venus, et aussi de l'impression d'avoir affaire à des gens qui se sont trop longtemps accommodés de la dictature. L'UPI, pour encadrer le plus d'émigrés possible, essaie donc de démontrer à ses militants, et aux lecteurs de son journal, que quelque chose est possible dans cette direction.

13 Les réticences sont plus manifestes en 1938 lors de l'arrivée des victimes des lois antisémites mussoliniennes (De Felice, 1961 ; Bonucci, 1998 ; Vial, 2000), juifs étrangers ou dénaturalisés expulsés d'Italie alors que celle-ci était après 1933 une destination certes peu recommandable au plan politique mais plus accueillante que la France républicaine (Hausman, 1995 ; Voigt, 1985). On constate pour le moins un désintérêt de la masse des antifascistes pour ces nouveaux arrivants, qu'il soit regretté et utilisé dans la polémique contre les autres groupes comme par Giustizia e Libertà pour qui l'antifascisme proteste "mais avec le ton et l'esprit de l'expédition des affaires courantes", ou réendossé et justifié, comme par un républicain mazzinien qui écrit : «Je dois avouer que parmi les antifascistes les mesures hitlériennes prises contre les juifs italiens ne font pas grande impression. Nous étions habitués à voir les juifs parmi les soutiens du fascisme. Dans la fureur anticatholique et antijuive des fascistes actuels, les ouvriers voient la vengeance de l'histoire, prédite et presque attendue » Les réformistes n'en sont peut-être pas loin lorsque leur journal note que les arrivants suivent un itinéraire que d'autres ont déjà connu mais en raison de choix politiques ${ }^{33}$. De fait, même si se crée à Paris une branche italienne de la LICA, elle est peu active, et laisse plutôt indifférente la masse des émigrés ${ }^{34}$.

14 Certes, des formes de réprobations s'expriment, autour du thème du retour au MoyenÂge ${ }^{35}$, avec une grande attention portée aux dramatiques errances alpines ou maritimes consécutives aux expulsions, dans la Voce degli italiani, et aussi dans les autres journaux malgré leur périodicité moins importante et leur peu d'intérêt pour les faits divers habituels, de moindre portée ${ }^{36}$. De même, des cas de suicide sont rapportés ${ }^{37}$. Et tous les groupes prennent des positions fermes ${ }^{38}$. L'UPI fait appel au sentiment national en présentant le racisme comme pur produit d'importation, et en rappelant de grands noms du Risorgimento ${ }^{39}$. Elle y ajoute l'intérêt national, la ruine de positions italiennes en Méditerranée orientale, la réduction du pays " au niveau d'une tribu de troglodytes " par les mesures contre les intellectuels ${ }^{40}$, Elle réussit même à revenir à la ligne de réconciliation nationale en soulignant le refus du racisme ou le piétisme de fascistes et plus encore de catholiques, en insistant sur des liens entre antisémitisme et 
anticatholicisme ${ }^{41}$. On peut se demander si l'objectif n'est pas double : faire condamner l'antisémitisme fasciste par un public imprégné de la judéophobie catholique, et, face aux militants, leur faire accepter des accommodements avec l'Église. Cela entre dans une stratégie populiste passant par la répudiation de l'anticléricalisme, mais on a une attitude assez comparable à Giustizia e Libertà ${ }^{42}$,

loin de cette ligne, et on retrouve la même attitude chez les réformistes ou les républicains ${ }^{43}$.

15 Reste que ce discours semble destiné à convaincre des militants réticents. Et il n'est pas exempt de dérapages. Certains relèvent de stéréotypes antisémites à base de richesse et de banque, chez les maximalistes et les républicains ${ }^{44}$ dont les imaginaires sont fort tributaires de la fin du XIX ${ }^{\mathrm{e}}$ siècle et que la situation d'exilés ne met pas à l'abri d'un nauséabond air du temps. Sourd aussi l'idée déjà rencontrée d'une justice immanente frappant des gens qui auraient ménagé ou soutenu Mussolini ${ }^{45}$, ce qui n'est pas faux pour des cas individuels mais tourne à l'accusation collective, raciste ${ }^{46}$. S'ajoute la tentation d'ironiser sur des gens devenus victimes de leur propre camp ${ }^{47}$. En mars 1939, la Voce degli italiani, qui a prôné le meilleur accueil pour tous ceux qui quittent l'Italie quelles qu'en soient les raisons, émet des réserves vis à vis de cette "nouvelle émigration » et demande des manifestations de soutien, mais ajoute qu'il «faut que nous soyons bien attentifs à ne pas tomber dans une position raciste en leur attribuant de façon exagérée aides et sympathie » et que "nous ne pouvons et nous ne devons pas étendre notre sympathie à ceux qui ne trouvent au fascisme aucun autre défaut en dehors de l'antisémitisme et qui conservent une position réactionnaire malgré notre attitude accueillante et notre propagande $»^{48}$. Ce type de commentaire, lié à un durcissement communiste, reflète une réaction courante, relevant moins de l'antisémitisme que d'une indifférence ou d'une inquiétude face à de nouveaux arrivants, d'autant que sous Daladier des décrets imposent des restrictions aux immigrés, et que l'inquiétude grandit. Il faut ajouter qu'une volonté de rectification existe, dans le cadre même du durcissement indiqué, avec à l'été une dénonciation réitérée de l'antisémitisme sur la base de celle des intérêts capitalistes, lecture sommaire mais efficace face aux militants ${ }^{49}$.

Sans ignorer l'antisémitisme hérité ou ambiant, les réactions aux arrivées de 1938 semblent correspondre avant tout à l'inquiétude d'un groupe qui se sent à peine installé, ou en difficulté, face à des nouveaux venus le ramenant à sa situation initiale en annulant ce qu'il a pu conquérir de façon concrète ou symbolique. On est d'une certaine façon dans la même situation qu'en 1930, car les nouveaux venus sont des inconnus pour la strate précédente, ceux qui ont une réelle notoriété l'ayant acquise dans l'Italie officielle ce qui ne plaide guère pour eux. Pour trouver une situation différente et des résultats non moins différents, il faut remonter juste après le milieu des années 1920, lors de la structuration de ce groupe.

\section{6-1927 : une inversion originelle, la victoire des derniers arrivés}

Divers idéologiquement, l'antifascisme l'est aussi par sa constitution. À l'origine, il rassemble quelques vieux émigrants installés dès avant 1914, ayant créé des sections socialistes ou républicaines, des migrants politisés mais qui ont quitté l'Italie pour des raisons économiques, des militants forcés de quitter leur pays par la violence fasciste avant la marche sur Rome ou peu après. Ni les rapports de police, ni une presse encore 
embryonnaire ne semblent permettre d'examiner les rapports entre eux et de mettre au jour d'éventuelles tensions. Ils tentent de s'organiser, mais les structures spécifiques manquent, et la LIDU, créée à Paris en 1923, n'est encore qu'un bureau d'aide juridique, vivotant sous la protection de sa consoeur et tutrice, la Ligue française, même si certains de ses responsables rêvent de remplacer les partis, réputés moribonds. C'est de cas de Campolonghi, émigré de 1898 (coll, 1989 ; Milza, 1981). Fort de ses contacts à Paris où il a été durant la guerre le représentant officieux de Leonida Bissolati, alors ministre, il contrôle une "page italienne" dans La France de Nice et du Sud-Est qui inquiète les autorités transalpines (Gastault, 2001) ${ }^{50}$, et est implanté dans le Sud-ouest agricole, où il lance un journal, Il Mezzogiorno, lié à la CGT et à l'anarcho-syndicalisme à travers De Ambris ${ }^{51}$. À côté de stratégies qui les mènent à prendre la direction de la LIDU, à la dynamiser et à l'engager plus clairement en politique, De Ambris et Campolonghi lancent début 1926 un débat portant en principe sur la façon d'agir contre le fascisme depuis l'extérieur. Comme chez d'autres outsiders (Bobbio, 1990: 174; Tranfaglia 1968 : 196), l'idée est de créer une structure extérieure aux partis existants : "les situations nouvelles n'ont jamais dans l'histoire été résolues par des hommes anciens" quels qu'en soient les mérites, que l'on prend soin de vanter ${ }^{52}$. Puis, quand les lois «fascistissimes» sonnent le glas de toute activité politique libre en Italie, les partis sont donnés pour morts, à remplacer par des « élites » agissantes ${ }^{53}$.

18 Mais les positions de Campolonghi sont trop peu solides pour faire l'unité de l'émigration autour de lui ${ }^{54}$, et son attachement à la démocratie parlementaire implique un raisonnable ralliement aux partis politiques dès que ceux-ci donnent signe de vie : en août, il salue un premier congrès en exil en écrivant que «le parti républicain nie notre présupposé par la méthode expérimentale. Il nous prouve le mouvement en marchant». Puis mi-octobre accueillant dans le Sud-ouest une réunion de républicains et de socialistes, il accepte l'enterrement des rêves de structures nouvelles au profit d'une coordination encore floue : il veut croire qu'elle rassemble des individus et non des représentants de partis, mais elle débouche sur un cartel, la Concentration antifascistes ${ }^{55}$. Surtout, la fin de toute vie politique en Italie entraîne l'exil des principaux dirigeants d'opposition, et il salue leur arrivée, la résurrection de leurs journaux, le fait qu'ils stimulent la ferveur des militants ${ }^{56}$. On est alors dans une situation inverse de celle des épisodes ultérieurs; les nouveaux venus, en position de force, absorbent et structurent ce qui existait avant eux.

Cela dit, la fusion n'est pas immédiate. On trouve encore trace de tensions début 1927. Campolonghi remet en avant des projets dépassant une alliance entre partis, ce qui permettrait à des personnalités extérieures, dont lui-même, de s'imposer. La police française l'imagine d'ailleurs capable d'organiser les exilés autour de lui, au moins en pays niçois. Il est poussé par De Ambris, qui ironise sur la multiplication des partis et prône l'unité dans la LIDU, ou par Silvio Trentin, ancien professeur de Droit et député libéral en exil (Rosengarten, 1980) ${ }^{57}$. Celui-ci pose le problème en termes de strates dans l'émigration. Il explique que l'inquiétude monte en province car les nouveaux arrivés se concentrent à Paris, et que s'il est normal que la capitale attire les hommes politiques professionnels, elle n'est pas toute la France; ceux qui s'y installent courent donc le risque de s'y isoler. Campolonghi affirme travailler "pour l'unité contre la scission » et peut ainsi se poser en interprète des émigrés de la France méridionale ${ }^{58}$. De plus, la désorganisation initiale de l'émigration a favorisé des sociabilités locales prenant le pas sur les partis, d'où les enthousiasmes unitaires à la base et les engagements dans des 
structures transversales. Des leaders illustres mais lointains, arrivés ensuite ne peuvent pas toujours repolariser les intérêts au bénéfice de leurs groupes spécifiques (Di Lembo, 1982: 229-230). Cela dit, il y parviennent le plus souvent, et même De Ambris doit admettre que "de l'union des vieux condottieri peut jaillir l'étincelle qui déclenchera l'action ${ }^{59}$. Les tensions entre strates de l'émigration politique se résolvent ainsi assez vite. La première était trop composite, la seconde trop riche de grands noms et la partie, au total, trop inégale...

20 Apparu avant la recomposition au bénéfice des partis, un quotidien, le Corriere degli italiani, lancé début 1926, reste quelque temps le témoin de ces velléités (Tobia, 1986). On y retrouve par définition des émigrés d'avant les lois fascistissimes, autour d'un des plus récents d'entre eux, Giuseppe Donati, ancien directeur du journal du Parti Populaire Italien, démocrate-chrétien, en pointe lors de l'affaire Matteotti, mais aussi un ancien consul à Dijon, propriétaire du titre de ce qui était jusque-là un hebdomadaire incolore. Ceux de ses collaborateurs ralliés à la Concentration antifasciste s'en écartent vite, et il devient le porte-voix des adversaires du cartel. Abandonné par Donati lui-même début 1927, le journal connaît une étrange dérive, entre difficultés économique, paradoxale proposition de vente à l'ambassade d'Italie, appuis de l'industriel turinois Riccardo Gualino à travers la banque Oustric, et surtout pour ce qui nous concerne ici, il multiplie les invectives contre les "généraux battus", des appels à la relève des générations ${ }^{60}$, et se constitue en organe d'une "anticoncentration » virtuelle à l'usage des mécontents et des isolés. Le ton est des plus aigres, contre la LIDU accusée de ne plus vouloir défendre les émigrés restés monarchistes ou de ne rien faire lorsque la police française perquisitionne le journal en compagnie d'un représentant du consulat fasciste, d'où démentis et polémiques ${ }^{61}$, et de façon générale contre un cartel remis en cause à la fois comme représentant du passé, d'une Italie préfasciste à ne pas laisser renaître, mais aussi comme tard-venu en France et illégitime à ce titre quand ses leaders sont qualifiés de "nouveaux riches en politique $»^{62}$. Même après décembre 1927 et l'interdiction du journal par les autorités françaises, pour cause d'appel à l'assassinat de Mussolini, ce thème continue de courir chez les mécontents, y compris Donati, marginalisé par l'anticléricalisme des exilés. Ainsi, en 1929, il évoque une émigration qui fut solidaire "au moins jusqu'au jour où l'arrivée à Paris des actuels chefs de l'antifascisme concentré a fait revenir toutes les vieilles rancœurs et les vieilles questions de personnes, qui ont causé dans notre patrie la ruine de la démocratie, et ici, en exil, la tourmentent mortellement ${ }^{63}$.

Restent quelques autres problèmes, comme en témoignent les difficultés de sections de la LIDU face aux Italiens de sections-soeurs françaises. Ainsi, à Villerupt, en Meurtheet-Moselle, où les Transalpins sont majoritaires dans la population, les autorités locales menacent d'expulsion les inscrits à la LIDU. Pour les intéressés c'est une manœuvre de la section française et de leurs compatriotes y adhérant, tenus pour philofascistes ${ }^{64}$, accusation d'ailleurs peu compatible avec leur engagement. On peut imaginer une question « de boutique ", la crainte d'une hémorragie de cotisants, mais les liens entre les organisations, la possibilité d'attirer de nouveaux membres, les doubles appartenances autorisées comme sur la Côte d'Azur font qu'il il y a plus à gagner qu'à perdre dans l'opération - d'ailleurs dans les Savoie ou le Var, les sections françaises suscitent leurs homologues ${ }^{65}$. Ici, on en est loin. On parle de querelles privées entre commerçants concurrents ${ }^{66}$, mais il s'agit bien de divergences entre strates de l'immigration. Les Italiens qui ont adhéré à la ligue française avant la création de la LIDU n'étaient pas favorables au fascisme, mais se préoccupaient peu de lutter contre 
lui, considérant que leur vie était désormais en Lorraine et s'intégrant à la vie politique locale, peut-être en vue d'une naturalisation. Certains peuvent aussi rejeter des spécificités de la LIDU, son républicanisme, son adhésion à la Concentration antifasciste, ce qui renvoie par exemple à l'adhésion à la seule Ligue française de Maria Rygier, hervéiste passée à un libéralisme prudent via le militantisme pour l'intervention de l'Italie dans la guerre en 1915 (Rygier, 1946). D'où un conflit entre ceux qui rêvent de retour offensif en Italie et ceux qui craignent un remue-ménage attirant l'attention de l'administration et rappelant leur statut d'étrangers. Ce conflit prend des allures de feuilleton, avec reconstitution de la section italienne, puis en 1930, succès obtenu par la direction parisienne de la Ligue française, passage massif d'Italiens d'une section à l'autre, renforcement de cette dernière réputée jusqu'alors à peine tolérée et vivotant ${ }^{67}$, d'où un renforcement de l'aile réformiste, modérée, au détriment des anciens responsables locaux proches de De Ambris et des maximalistes, et d'infinies polémiques $^{68}$, sans même que les tensions avec la section française soient tout à fait résorbées ${ }^{69}$. Ce n'est certes qu'un épisode local et mineur, encore qu'on trouve ailleurs des traces montrant qu'il n'est pas isolé ${ }^{70}$, mais c'est un élément de la multiplicité des situations et des rapports entre migrants, y compris à l'intérieur d'une émigration politique et lorsque les questions idéologiques ne semblent guère en cause.

\section{Conclusion}

De façon générale, ces épisodes de tension, même s'ils ne constituent pas la trame de l'histoire de l'émigration politique italienne en France, se prolongent et de mêlent, finissant par occuper l'essentiel de la période - leur pérennité pourrait être un facteur permettant de plaider leur importance souterraine. Il faut ajouter qu'ils restent toujours à l'arrière-plan, d'autant qu'ils sont susceptibles de gêner à terme à peu près tous les protagonistes, surtout lorsqu'ils se sont soldés par une fusion partielle, une transaction implicite au terme de laquelle il convient d'oublier ou de refouler les difficultés initiales. Ils sont enfin difficiles à dégager d'autres problèmes, d'autres tensions, et leur aboutissement dépend de rapports de force réels ou symboliques, aux déterminations multiples et dont on vient de voir qu'ils n'étaient pas toujours défavorables aux derniers venus.

Cela dit, il ne saurait s'agir ici que d'une esquisse, à partir d'un seul cas et d'exemples trop vite présentés. De multiples comparaisons seraient nécessaires, à travers l'espace et le temps. On pourrait imaginer de trouver des ressemblances et des différences entre l'arrivée des leaders antifascistes en France en 1926 et la tentative de prise en main des organisations grecques par les exilés politiques d'après 1967, ou ajouter un cas de figure absent dans le cas étudié, celui du conflit entre vagues constituées, structurées, ayant des légitimités politique comparables mais incompatibles, comme les exilés des pays de l'est partis dès les lendemains de la Seconde Guerre mondiale et ceux qui ont voulu agir dans le cadre des démocraties populaires, et les ont fuies après l'écrasement de la révolution hongroise (Paillot, 1990 : 59-60) ou du printemps de Prague (Dufoix, 2002). Il y aurait là un chantier à ouvrir, à condition sans doute de toujours garder à l'esprit qu'il s'agit d'un élément et d'un élément seulement, certes souvent oublié, dans la complexité du réel d'émigrations elles mêmes particulières... 


\section{BIBLIOGRAPHIE}

BOBBIO Norberto (1990) Profilo ideologico del ‘900, rééd. Milan, Garzanti, 324 p.

COLLECTIF (1981) Luigi Campolonghi Une vie d'exil (1876-1944), Paris, CEDEI, 102 p.

DE FELICE Renzo, (1961) Storia degli ebrei italiani sotto il fascismo, rééd. 1993, Turin, Einaudi, XXXVI, $648 \mathrm{p}$.

DI LEMBO Luigi (1982) L'organizzazione dei socialisti italiani in Francia, in Coll., L'emigrazione socialista nella lotta contro il fascismo (1926-1939), Florence, Sansoni, pp. 221-261.

DROZ Jacques (1985) Histoire de l'antifascisme en Europe 1923-1939, Paris, La Découverte.

DUFOIX Stéphane (2002), Politiques d'exil, Paris, PUF, 318 p.

GAROSCI Aldo (1953) Storia dei fuorusciti, Bari, Laterza, 314 p.

GASTAUT Yvan, (2001), La France de Nice et du Sud-Est. Journal événement (1926-1928), Cahiers de la Méditerranée, VI, pp. 175-182.

HAUSMAN Frank-Rutger (1995) Le tappe dell'esilio : studiosi tedeschi in Italia, in Etappen. Deutsche Wissenschaftler in Italien in Rifugio Precario - Zuflucht auf Widerruf. Artisti e intellettuali in italia, 1933-1945, Deutsche Künstler und Wissenschafter in Italien, Berlin/Milan, Akademie der Künste/ Mazzotta pp. 175-182 et 191-198.

MATARD-BONUCCI Marie-Anne (1998) L'antisémitisme en Italie : les discordances entre la mémoire et l'histoire, Hérodote, 2, pp. 217-238.

MILZA Pierre (1988) «Émigrés politiques» et «Émigrés du travail» italiens en France d'après le fonds du Casellario politico centrale, Mélanges de l'école française de Rome-Moyen-âge/Temps modernes, 1, pp. 181-186.

MILZA Pierre (1988-2) L'intégration des Italiens en France : « miracle » ou vertus de la longue durée, Pouvoirs, $n^{\circ}$ 47, pp. 104-113.

MILZA Pierre dir. (1986) Les Italiens en France de 1914 à 1940, Rome, ÉfR, VIII, 788 p.

MILZA Pierre (1981), Français et Italiens à la fin du XIXe siècle, Rome, ÉfR, 1114 p.

PAILLOT Patricia (1990) La communauté grecque de Toronto, thèse, Bordeaux 3.

RAPONE Leonardo (1986), I fuorusciti italiani, la seconda guerra mondiale e la Francia, in Pierre Milza (dir.), Les Italiens en France de 1914 à 1940, Rome, ÉfR, pp. 343-284.

RAPONE Leonardo (s. d.) L'Union populaire italienne, in (Coll.), L'Italia in esilio. L'emigrazione italiana in Francia tra le due guerre. L'Italie en exil. L'émigration italienne en France entre les deux guerres, Rome, Presidenza del Consiglio dei ministri, Dipartimento per l'Informazione e l'Editoria, pp. 338-340.

RYGIER Maria (1946), Rivelazioni sul fuoruscitismo italiano in Francia, Rome, SRR, 64 p.

SORI Ercole (1979), L'emigrazione italiana dall'unità alla seconda guerra mondiale, Bologne, Il Mulino, $305 \mathrm{p}$.

TÉMIME émile (1991), Émigration « politique » et émigration " économique », in (Coll.)

L'émigration politique en Europe aux XIXe et XXe siècles, Rome, ÉfR, pp. 52-72. 
TOBIA Bruno, Il Corriere degli italiani : la parabola di un quotdiano antifascista in francia, in Pierre Milza (dir.), Les Italiens en France de 1914 à 1940, Rome, ÉfR, pp. 285-321.

TOMBACCINI Simonetta (1988) Storia dei fuorusciti italiani in Francia, Milan, Mursia, X, 380 p.

TRANFAGLIA Incola (1968), Carlo Rosselli dall'interventismo a Giustizia e Libertà, Bari, Laterza, $390 \mathrm{p}$.

URBAN Joan-Barth (1986) Moscow and the italian comunist party - from Togliatti to Berlinguer, Londres, Tauris, $370 \mathrm{p}$.

VIAL Éric (2001) Populisme et communisme, l'Union Populaire Italienne, une organisation de masse du PCI en exil (1937-1940), Laboratoire italien, 1, pp. 99-108.

VIAL Éric (2000) Les Antifascistes italiens en exil en France face aux lois antisémites mussoliniennes de 1938, Cahiers de la Méditerranée, nº 61, pp. 227-245.

VIAL Éric (1999) L'Unione popolare italiana (Upi) 1937-1940. Un'organizzazione di massa comunista in esilio, Mezzosecolo ${ }^{\circ} 12$, XII, pp.

VIAL Éric (1998) La Ligue française des Droits de l'Homme et la LIDU, son homologue italienne, organisation d'exilés antifascistes dans l'entre-deux-guerres, Le Mouvement social, $\mathrm{n}^{\circ} 183, \mathrm{pp}$. 119-134.

VIAL Éric (1988) Émigrés politiques, immigrés qui se politisent : quelques données tirées des dossiers du Casellario politico centrale (Rome)», Mélanges de l'école française de Rome - Moyen-âge/ Temps modernes, 1, pp. 73-93.

VIAL Éric (1986) La Ligue Italienne des Droits de l'Homme (LIDU), de sa fondation à 1934, in Pierre Milza (dir.), Les Italiens en France de 1914 à 1940, Rome, ÉfR, pp. 407-430.

VOIGT Klaus (1985), Gli emigrati in Italia dai paesi sotto la dominazione nazista : tollerati e perseguitati 1933 1940, Storia contemporanea, 1.

ROSENGARTEN Frank (1980) Silvio Trentin dall'interventismo alla Resistenza, Milan, Feltrinelli, 244 p.

\section{Presse : publications citées}

Giustizia e Libertà

Il Grido del popolo

Il Nuovo Avanti

Il Pungolo

Il Risveglio Anarchico

L'Adunata dei Refrattari

L'Avanti

La France de Nice et du Sud-Est

La Giovine Italia

La Lega

La Libertà

La Voce degli italiani 
La Voce Nuova

L'Avvenire del Lavoratore

Le Petit Niçois

L'Esilio

\section{NOTES}

1. Archives Nationales, Paris, (AN), F7 13251, 10 I 1931 ; Archives des Affaires étrangères, Paris, Europe 30-40, (AQO), Italie 254, Vintimille 14 III 31, Menton 3 VI 1932.

2. L'Avanti (AV) XI 1930, «Perché la Francia respinge la nuova emigrazione italiana ».

3. AQO, Italie 243, Beausoleil 3 XI 1930, Menton 5 XI 1930, Paris 8 XI 1930.

4. AQO, Italie 254, Beausoleil 3 X 1930; La Libertà (Lib) 30 VIII 1930, 1 I 1931; L'Avvenire del Lavoratore (Avv) 25 X 1930 ; AV, XI 1930 ; AN, F7 13251, 10 I 1931.

5. Lib 9 et 23 VIII, 10, 17 X, 7 XI 1930 ; Archivio Centrale di Stato, Rome, (ACS), SPD CR 77, Paris 16 XII 1930.

6. Lib 9 VIII, 20 IX, 5, 25 XII 1930 ; Avv 15 XI 1930.

7. Lib 5 ; 25 XII 1930, 5 II, 15 X 1931, Avv 10 X 1931 ; La Voce Nuova 15 X 1931 ; Il Risveglio Anarchico 17 X $1931 ; A V 18$ X 1931.

8. ACS, CPC 1101, Trevise 22 VI 1932, Rome 1 IV 1932, Paris 27 V 1932 ; ACS, CPC 5487, Chieti 28 XII 1938, Nancy 5 VIII 1938; ACS, CPC 5485, Nice 30 III 1939. Par ailleurs sur un échantillon constitué par l'École française de Rome et comprenant $5 \%$ des dossiers individuels établis par la police politique fasciste sur des Italiens passés par la France, quelque 120 sont arrivés en 1930, on peut supposer qu'ils sont plus de 2000 dans ce cas à avoir attiré l'attention du régime, ce qui, sur quelque 20000 dossiers, n'est pas négligeable.

9. Lib, 26 VII 1930

10. Les Cahiers des droits de l'Homme (CDH) 1929, p. 613.

11. Lib. 30 VIII, 6, 13 IX 1930, ACS G1 234, Rome 7 IX 1930.

12. Par ex. AQO Italie 354, Tunis 8 XI 1930, AV. XI 1930.

13. ACS CPC 1632, 18 X 1930 ; L'Esilio 16 XII 1930.

14. Le Petit Niçois $2 \mathrm{X} 1930$.

15. ACS CPC 1632, doc. cit. ; ACS G1 235, s.l.n.d. [XII 1931] ; Lib 19 XI 1931.

16. AQO Italie 254 Bordeaux $27 \times 1930$.

17. ACS AGR 1932 200, Rome 22 IX 1932.

18. Lib 20 VIII 1931.

19. L'Esilio 16 XII 1930, 1 I 1931 ; ACS G1 235, Chambéry 18 III 1931.

20. Lib 19 IX 1931.

21. ACS G1 235, XII 1931; ACS PP 26-3, Lyon 14 XII 1931, Paris 19 XII 1931 ; La Lega 15 IV 1932.

22. Lib 11 VIII 1932.

23. Voir Palmiro Togliatti, Appel aux fascistes, Paris, Nautilus, 1983, avec une présentation très polémique.

24. Voir par ex. Il Grido del popolo 14 XI 1936, 13 II 1937 ; La Voce degli italiani (Voce) 5 IX 1937, 21 I 1938.

25. Unione Popolare italiana, Congresso di Lione 18-19 Marzo 1937, Paris, UPI, 1937.

26. Fondazione Gramsci, Rome, Archivio del Partito Comunista (APC) 1349-1, II 1936 ; ACS AGR 1937 38, Paris 18 III 1937.

27. ACS AGR 1937-68, Paris 13 I 1937 ; APC 1440-1, Paris 21 I 37 ; Voce 3 VI 1937.

28. Voce 14 X, 16 XII 1937, 16 VII 1938, 28 II, 12 III, 23 V 1939. 
29. ACS AGR 1937 38, s l n d ; Voce 21 VIII 1937, 8 V 1938.

30. Voce $24 \mathrm{X}, 9$ XI 1937.

31. Voce 8 II, 20 VII, 13 VIII 1938.

32. Voce 4, 11 VI 1939.

33. GL 26 VIII 38 ; La Giovine Italia (GI ) 10 IX 1938 ; NA 25 III 39.

34. Voir par ex. ACS CPC 3961, Lyon 3 VIII 1939.

35. GI 10 IX 1939, GL 22 VII 1938, 7 VII 1939, NA 10 IX, 3 XII 1938, AV 11 XII 1938, Voce 2 IX, 3 XI 1938, etc.

36. Voce 10, 14, 16, 17, 21, 24, 25, 26, 28 III, 6, 7, 13 IV, 4, 10 V, 8, 13 VII, 3, 11, 12, 16, 24, 26 VIII 1939 ; GI 28 I, 22 IV 1939 ; GL 17 III, 16 VI 1939 ; NA 17 IX 1938, 25 III 1939, etc.

37. Voce 10 XII 1938, 12 VIII 1939 ; GI 17 XII 1938, 18 II, 22 IV 1939 ; NA 4 II, 22 IV, 17 VI 1939 ; GL 17 II, 17 III 1939.

38. GI 10 IX 1938, 17 VI 1939 ; L'Adunata dei Refrattari 3 IX 1938, 10 VI 1939 ; AV 10 IV 1938 ; Lo Stato operaio 1 XII 1938, p. 361 ; Voce 18 III 1939; GL 18 XI 1938.

39. Voce 2, 27 I, 22 VI, 14, 16, 17, 24 VII, 4, 10, 30 VIII, 8, 17, 27 IX, 3, 12, 15, 17, 19 XI, 3, 4 XII 1938, 17 I, 7 II, 10 V 1939, etc.

40. Voce 20, 7 IX, 10, 18 X, 19 XI, 10, 29 XII 1938, 28 III, 6 IV, 3, 4, 7 I, 20 V 1939.

41. Voce, 2 I, 13 VIII 10, 16 IX, ,9, 16 X, 8, 16, 22 23, 26, 29 XI, 3, 17, 8 XII 1938, 19 I, 2, 25, 28 II, 3 VIII 1939 ; ACS PPM 107, Paris 22 VIII 1938.

42. GL 29 VII, 30 IX 1938, 13 I, 3 II 1939, etc.

43. NA 19 XI 1938, 17 VI 1939 ; GI 16 VII, 10, 17 IX 1938, 22 IV 1939.

44. GI 22 I, 5 II, 16, 23, 30 VII, 3, 10 IX, 23 X, 3 XII 1938, 21, 28 I, 25 II, 20 V, 3 VI 1939 ; AV 31 VII, 11 XII 1938.

45. GI 23 VII, 20 VIII, 3, 10, 17 IX 1938; GL 11, 18 II, 23 IX, 14 X 1938 ; NA 10 IX, 29 X, 3 XII 1938 ; AV 31 VII 1938 ; Voce 22, 29 III 1939.

46. GI 26 XI 1938.

47. NA 17 IX, 22, 29 X 1938, 25 III 1939.

48. Voce 17 III 1939.

49. Voce 23, 29 VI, 2, 12 VII, 11, 20 VIII 1939.

50. ACS CPC 988, Consul Nice 15 II 1927 ; AN F7 13460, 3 XII 1926, 3 II, 18 III, 8 IV 1927 ; I Documenti Diplomatici Italiani, $7^{\mathrm{e}}$ série, Rome, Libreria dello Stato, (DDI), V, 1967, p.3, Rome 7 II 1927 .

51. ACS CPC 1690, biographie; AN F7 13000, Agen 4 XII 1925 ; AN F7 13450, Toulouse 2 I 1927, Ministère italien des Affaires étrangères, Rome, (AAE), 85-2, Rome 14 X 1925.

52. Il Mezzogiorno (Mezz) 23 I, 6 II 1926; Il Corriere (Corr) 9 IV 26; La France de Nice et du Sud-Est (FNSE) 5 IV 1926.

53. FNSE 17, 21 VI 1 VII 1926.

54. Par ex ACS CPC 988, Nice 30 VII 1926.

55. FNSE 24 VIII, 22 X 1926

56. FNSE 8, 9, 17 XII 1926, 13 I 1927.

57. FNSE 25 I 27 ; Mezz 5, 12 III 1927 ; R. De Felice, «Il partito repubblicano nell'emigrazione antifascista ", La Voce repubblicana, 4-5 juin 1966 (Pavie 28 I 1927) ; ACS CPC 988, s.1. 23 II 1927 ; ACS AGR 1927 147, s.l. 4 III 1927.

58. FNSE 19 XII 1926.

59. Mezz 19 III 1927.

60. Corr 17, 28 IV 1927,

61. FNSE 19 VI, 14, 20 VIII 27, Corr 6, 14 VIII, 14, 22 X 1927 ; ACS G1 233, Mulhouse 28 X 1927 ; Lib $23 \times 27$.

62. Corr. 13 V 1927, 27 IX 1927 "Quello che tutti i giovanni sentono... ».

63. Il Pungolo, $15 \mathrm{~V} 1929$.

64. Lib 10 III 1929 ; ACS G1 234, 14 VI 1929. 
65. ACS CPC 1632, Nice 7 VIII 1929 ; ACS CPC 4405, Nice 19 VI 1929 ; ACS G1 234, Nice 2 II 1929 ; Lib 13 XI 1927, 14, 21 X 1928, 17 IV, 10, 17 V, 25 XII 1930 ; Avv 24 V 1930 ; Corr 25 IX 1926.

66. $C D H 1929$, p. 716.

67. Lib 17 III, 30 VI, 14 VII, 18 VIII 1929 ; ACS CPC 1632, Paris 29 I 1929 ; ACS G1 239, Nancy 10 IV 1930.

68. Lib 5, 12 II 1931, 11 II, 28 VII, 3 XI 1932, 23 II 1933 ; AV 8, 29 III, 10 V, 14 VI, 5, 12 VII 1931, 31 I, 21 II 1932, 4 III 33 ; Avv 14 III, 4 VII, 15 VIII 1931, 7 V 32 ; ACS PP 32-9, Villerupt 17 I 1932 ; ACS CPC 1976, 7 IX 1931.

69. $C D H 1932$, p. 37, CDH 1933, p. 376.

70. Voir par ex. pour Tucquegnieux, également en Meurthe-et-Moselle, Lib 15 II 1930.

\section{RÉSUMÉS}

À côté d'oppositions régionales, idéologiques, ou entre émigrés politiques et économiques, d'autres sont "générationnelles ", entre vagues d'arrivée, même séparées par peu d'années. Dans le cas italien, le prototype est 1930, quand Mussolini rouvre la frontière : des migrants à peine installés se sentent menacées par les arrivées, les nouveaux venus ont accepté trop longtemps de vivre sous la dictature et sont suspects ; certains les défendent, il y a débat mais l'émigration non communiste a pour le moins du mal à les recevoir et les accepter. La conscience de ces problèmes n'est pas le moteur de l'attitude du PCI quelques années plus tard, et même si la tactique de la main tendue rend les contacts plus faciles, les vieux militants ne sont pas convaincus. En 1938 les victimes des lois antisémites mussoliniennes ne sont pas toujours très bien vues. Il y a un problème de légitimité politique, illustré dix ans plus tôt avec un résultat inverse, quand les nouveaux venus, responsables de partis, ont subordonné les premières organisations spontanées, malgré le maintien de structures dissidentes. Ces exemples de tensions, le plus souvent refoulées et oubliées, peuvent être des éléments complémentaires dans notre lecture de l'histoire des migrations politiques.

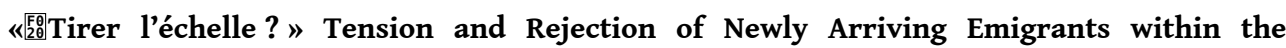
Antifascist Italian Emigration in France. An addition to regional and ideological oppositions, or those between political and economic emigrants, other oppositions are 'generational', between groups arriving at different times, even when their arrival is separated by only a few years. In the case of Italy, the prototype is 1930 when Mussolini re-opened the border; migrants who had just settled in France felt threatened by the new arrivals, for the latter, having accepted for too long to live under the dictatorship, were suspect. Certain defended them, discussion takes place, but the non communist emigration had difficulty receiving and accepting them. Acknowledgement of these problems is not behind the attitude of the PCI (Italian Communist Party) several years later, and even if the tactic of extending a welcome eases the contact between the groups, the older militants are not convinced. In 1938 the victims of Mussolini's anti-Semitic laws are not always well-received. There is a problem of political legitimacy, illustrated ten years earlier by the inverse example, when the new arrivals, Party leaders, subordinated the first spontaneous organisations despite the maintaining of dissident structures. These examples of tensions, most often forgotten or repressed, might serve as complementary elements in our reading of the history of political migrations. 
"Tirer l'échelle?» Tensiones y rechazos de nuevos inmigrantes en el seno de la emigración antifascista italiana. Junto a oposiciones regionales, ideológicas o entre emigrantes políticos y económicos, cohabitan las oposiciones "generacionales » entre olas de emigración, incluso cuando la separación entre éstas es de pocos años. En el caso italiano, el prototipo es 1930, año en el que Mussolini reabre las fronteras: emigrantes apenas instalados se sienten amenazados por los recién llegados, que han aceptado vivir bajo la dictadura durante demasiado tiempo y que, por lo tanto, resultan sospechosos. Algunos los defienden, surge el debate, pero a la emigración no comunista le cuesta menos recibirlos y aceptarlos. La conciencia de estos problemas no es el motor de la actitud del PCI años más tarde, e incluso, aunque la táctica de la mano tendida vuelve los contactos mas fáciles, los viejos militantes no están convencidos. En 1938 las víctimas de las Leyes antisemitas de Mussolini no están tampoco siempre bien vistas. Hay un problema de legitimidad política, ilustrado diez años atrás por un resultado inverso: los recién llegados responsables de partidos controlaron a las primeras organizaciones espontáneas, a pesar de la persistencia de estructuras disidentes. Estos ejemplos de tensiones, en la mayoría de los casos rechazados y olvidados, pueden aportar elementos complementarios a la lectura de la historia de las migraciones políticas.

«Tirer l'échelle?» Tensioni e rigetti degli ultimi arrivati, nell'emigrazione antifascista italiana. Accanto a opposizioni regionali, politiche o fra emigrati economici e politici, altri sono " generazionali ", fra ondate, anche separate da solo pocchi anni. Nel caso italiano, il modello é il 1930, colla riapertura della frontiera da Musossolini : emigranti appena sistemati provano un'impressione di minaccia dagli arrivi, gli ultimi arrivati hanno troppo a lungo accettato di vivere sotto la ditattura, sono sospetti. Hanno qualche difensore, c'é un dibattito, ma l'emigrazione non-comunista a per lo meno difficoltà a accoglierli ed accettarli. La coscienza di questi problemi non é il motore dell'atteggiamento del PCI alcuni anni dopo, ed anche se la tattica della «mano tesa » facilita i contatti, vecchi militanti non sono convinti : nel 1938, le vittime delle leggi razziste mussoliniane non sono sempre ben considerati. C'é un problema di leggitimitù politica, illustrato dieci anni prima con un sito opposto, quando gli ultimi arrivati, esponenti di partiti, hanno subordinato le prime organizzazioni spontanee, anche se stutture diissdenti si sono mantenute. Questi esempi di tensioni, per lo più rimote e dimenticate, possono essere elementi complementari nelal nostra lettira della storia delle migrazioni politiche.

\section{INDEX}

Mots-clés : asile politique, histoire, Italiens, relations intra-communautaires

\section{AUTEUR}

\section{ÉRIC VIAL}

Professeur d'histoire contemporaine, Université Pierre Mendès-France (Grenoble 2), UFR

Sciences Humaines, 1281 avenue centrale, Domaine Universitaire, BP 47, 38040 Grenoble cedex 9, France. E-mail : vialeric@club-internet.fr 